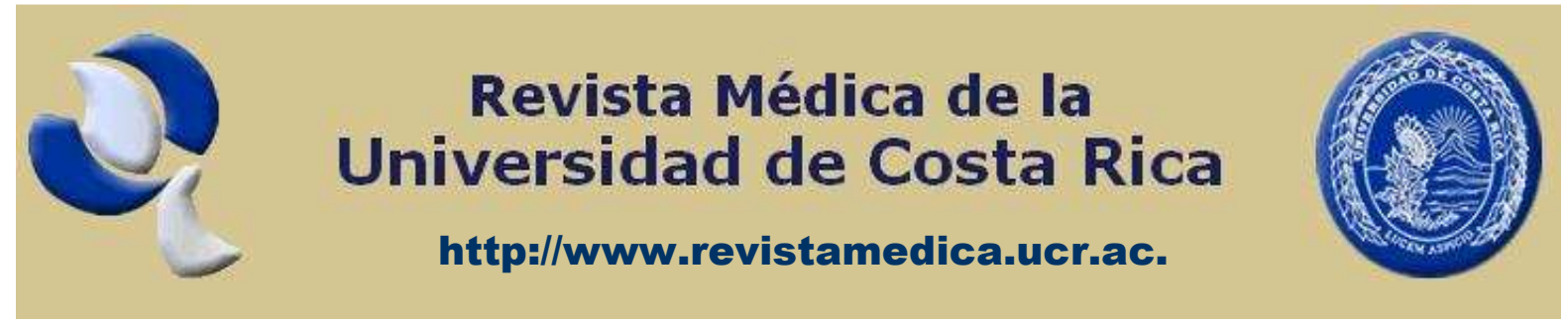

Investigación original

\title{
Valoración de la prescripción de antibióticos en pacientes internados en la Unidad de Cuidados Intensivos de un Hospital Privado en Costa Rica diagnosticados con neumonía.
}

\author{
Rodríguez Berrocal, Franklin'; Valverde Ríos, Marcela'; Zavaleta Monestel, \\ Esteban $^{2}$ y Chaverri Fernández, José Miguel ${ }^{3}$.
}

1. Estudiantes Internos de Farmacia. Universidad de Costa Rica, San Pedro, San José, Costa Rica. 2. Farmacéutico. Farmacia Hospital Clínica Bíblica, San José, Costa Rica. 3. Farmacéutico. Centro Nacional de Información de Medicamentos. Facultad de Farmacia. Universidad de Costa Rica, San Pedro, San José, Costa Rica.

Resumen: La neumonía es una infección pulmonar. Puede ser causada por diversas especies bacterianas, virus, hongos y parásitos. La identificación del microorganismo etiológico es de importancia para elección del tratamiento antimicrobiano adecuado. Se realizó un análisis de la estrategia antibiótica utilizada en la unidad de cuidados intensivos de un hospital privado en Costa Rica para pacientes ingresados por neumonía, ya sea adquirida en la comunidad o nosocomial. En los internamientos valorados se determinó que se realizaron varios cambios en la terapia antibiótica denotándose fallos en el seguimiento o conocimiento de los datos epidemiológicos y las sensibilidades bacterianas de los mismos, lo cual podría generar fallos en la terapia prescrita, deterioro del paciente, estancias hospitalarias mayores y aumento de la resistencia de las bacterias a los antibióticos. Es necesaria la introducción de procedimientos y suministro de información que establezcan un mejor manejo de este tipo de pacientes, su patología y todo lo relacionado a la terapéutica bacteriana a elegir dentro del hospital.

Palabras clave: Antibioticoterapia, neumonía, antibióticos, vías respiratorias, unidad de cuidados intensivos.

Revista electrónica publicada por el Departamento de Farmacología de la Escuela de 
Recibido: Junio 2008. Aceptado: Julio 2008. Publicado: Septiembre 2008.

Abstract: Pneumonia is a lung infection usually caused by several types of bacteria, virus or fungi. The identification of the etiological microorganism is very important to choose the best antibiotic therapy. We made an analysis of the antibiotic strategy applied in a group of community acquired pneumonia or nosocomial pneumonia patients of an intensive care unit in a private hospital in Costa Rica. During the treatment of these patients the physician made several changes of the antibiotic therapy, showing that there are several problems related with the knowledge of the epidemiology and sensitivity of the antibiotics used for this pathology in this hospital. This may cause therapeutically failures, increase patients side effects and longer hospitalization. The introduction of new procedures and information that can help improve the therapy with antibiotics for these patients, their pathologies and every rational-use related issues is necessary

Keywords: Antibiotic therapy, pneumonia, antibiotics, respiratory airways, intensive care unit.

\section{Introducción}

La neumonía es una infección del parénquima pulmonar. Puede ser causada por diversas especies bacterianas, micoplasmas, clamidias, rickettsias, virus, hongos y parásitos. La neumonía no es, por tanto, una enfermedad única, sino un grupo de inscripciones específicas, cada una con su epidemiología, patogenia, presentación clínica y evolución diferentes [1]. La identificación del microorganismo etiológico es de capital importancia ya que constituye la clave del tratamiento antimicrobiano adecuado. No obstante, debido a la naturaleza grave de la infección, muchas veces se precisa iniciar inmediatamente el tratamiento antimicrobiano antes de recibir la confirmación del agente causal por el laboratorio [1]. La aspiración de estos patógenos es el mecanismo causal más común. Desde el punto de vista del lugar de adquisición, las neumonías podrían clasificarse en comunitarias y hospitalarias. A lo largo de los años y debido al aumento en el número de pacientes con estas características y al mejor conocimiento de sus particularidades, la neumonía ha ido adquiriendo más importancia en pacientes institucionalizados. En las últimas guías clínicas, estas neumonías se incluyen junto con las neumonías adquiridas en el hospital en un grupo común denominado neumonías relacionadas con el medio sanitario $[2,3]$.

Los pacientes con neumonía usualmente presentan tos (más del 90\% de los casos), así como aproximadamente presentan un aumento en la producción de esputo en un $66 \%$ de las ocasiones y dolor torácico pleurítico en un $50 \%$ [4]. En ocasiones la sintomatología no respiratoria pueden predominar [4].

Determinar la etiología de la neumonía permite plantear un tratamiento dirigido, cambiar racionalmente a la vía oral cuando se requiera, evitar el uso inadecuado de antibióticos y conocer la prevalencia y el patrón de sensibilidad de los patógenos. El inicio del

Revista electrónica publicada por el Departamento de Farmacología de la Escuela de 
tratamiento en las primeras ocho horas ha demostrado disminuir la mortalidad en pacientes de edad avanzada. En la población general otros estudios sugieren iniciar en las primeras cuatro horas desde su presentación clínica [2]. La incidencia de neumonía es superior en los pacientes de edad avanzada en relación a los más jóvenes, esta incidencia se multiplica según aumenta la edad de los individuos. Las circunstancias de vida del paciente, su ocupación, los antecedentes de viajes, exposición a animales o mascotas y el conocimiento de la curva epidemiológica de los brotes en la comunidad donde vive son datos útiles para descubrir la etiología microbiana de la infección [1].

De acuerdo con la etiología podría decirse de manera general que ciertos agentes se aíslan con similar frecuencia; dentro de los más comunes tenemos al Streptococcus pneumoniae; otros etologías más frecuentes pero en pacientes menos graves son los virus respiratorios, el Mycoplasma pneumoniae, la Chlamydophila pneumoniae. Algunos son más

\section{Resultados}

El presente trabajo se describe como un estudio retrospectivo observacional diseñado para valorar la prescripción de antibióticos de los pacientes internados en la Unidad de Cuidados Intensivos de un Hospital Privado diagnosticados con neumonía durante el período 20052006. El total de pacientes internados en este periodo fue de 64 , de los cuales 24 cumplían con los criterios establecidos por el estudio. La recolección de los datos asociados a estos pacientes se realizó con la ayuda del departamento de informática. Los criterios de inclusión fueron: mayores de frecuentes en situaciones más graves (Legionella pneumophila, bacilos gramnegativos aerobios, Staphylococcus aureus). La elección inicial del tratamiento antimicrobiano a menudo es empírica, se basada en el contexto en que se adquirió la infección, la presentación clínica, los tipos de anomalías presentes en la radiografía de tórax, la tinción del esputo u otros líquidos corporales infectados. Una vez identificado el agente etiológico se puede elegir un tratamiento antimicrobiano [1]. La recomendación del antibiótico debe hacerse frente a un diagnóstico clínico, basándose en datos epidemiológicos $y / 0$ antecedentes del enfermo que ayuden a predecir los patógenos blanco. Si bien algunos agentes tienen similar resistencia en todo el mundo (Moraxella catarrhalis, micoplasma, clamydia y legionella), en otros la situación varía localmente; por esto es importante realizar estudios regionales, adecuadamente diseñados que valoren la resistencia a estos y otros patógenos.

40 años, diagnosticados por Neumonía Adquirida en Comunidad (NAC) y/o nosocomial con secreciones purulentas acompañadas de fiebre después de 48 horas de internamiento, que se le halla realizado un cultivo o frotis de las secreciones bronquiales 0 aspirado bronquial y que tuvieran un resultado positivo para la presencia de bacterias. Se recopilaron todos los documentos y la información teórica necesaria para dar inicio a la investigación mediante la colaboración de la subdirección médica, los departamento de informática y registros médicos.

Revista electrónica publicada por el Departamento de Farmacología de la Escuela de 
La autorización para la realización de este estudio y la utilización de los expedientes electrónicos fue proporcionada por el Hospital con la autorización de la Dirección y Subdirección Médica de la institución, en ausencia aun en ese momento de un Comité Ético Científico dentro de este. Se revisó tanto el diagnóstico de ingreso como el de egreso, edad, sexo, comorbilidades, estancia hospitalaria, manifestaciones clínicas referentes a neumonía, utilización de ventilación mecánica, utilización de pruebas confirmatorias como radiografía de tórax ó cultivos de esputo ó aspirado bronquial, pruebas de sensibilidad a antibióticos, y la terapia antibiótica inicial así como los respectivos cambios subsiguientes, en caso de que se hubieran presentado. La población de 24 pacientes se encuentra conformada en su gran mayoría por personas mayores de 65 años, estos presentan una distribución por género mayor para hombres que para mujeres. De la población analizada solamente dos pacientes no presentaban otras patologías durante el período en estudio; la gran mayoría presentaban afecciones cardíacas, respiratorias e insuficiencia renal, lo cual es característico del grupo etáreo analizado. La mayoría de los pacientes tuvieron una estancia en el hospital de más de dos días, todos estuvieron como mínimo un día. El máximo de tiempo fueron 25 días para uno de los pacientes. El promedio de días de hospitalización fue de 8,2 días.

Las neumonías nosocomiales requirieron mayor cantidad de días de hospitalización que las neumonías adquiridas en la comunidad, adicionando que el $58,3 \%$ de los pacientes necesitaron ventilación mecánica. No se logra elucidar una relación clara entre la ventilación mecánica y la cantidad de días de internamiento.

\section{Cuadro $\mathbf{N}^{\circ} 1$ Tipo de neumonía diagnosticada por paciente}

\begin{tabular}{|c|c|c|}
\hline Patología & $\begin{array}{c}\text { Número } \\
\text { de } \\
\text { Pacientes }\end{array}$ & Porcentaje \\
\hline $\begin{array}{c}\text { Adquirida } \\
\text { en } \\
\text { comunidad }\end{array}$ & 14 & 58.3 \\
Nosocomial & 10 & 41.7 \\
\hline
\end{tabular}

Como método diagnóstico se utilizo en el $41,7 \%$ de los pacientes la radiografía de tórax, en el $50 \%$ de los pacientes se utilizó el cultivo de esputo o de aspirado bronquial como método diagnóstico de neumonía.

Ambas pruebas confirmatorias no son complementarias, pues en algunos pacientes se realizó tanto cultivo como radiografía de torax.

En el $54,2 \%$ de los casos no se especificó el patógeno, esto a pesar de realizarse en un $50 \%$ de los casos la prueba. De los casos en los que logró identificar al agente causal el más común, tanto sólo como acompañado de otros patógenos fue Pseudomona aeruginosa. Los antibióticos que se utilizaron con mayor frecuencia durante el inicio del

Revista electrónica publicada por el Departamento de Farmacología de la Escuela de Medicina de la Universidad de Costa Rica, 2060 San José, Costa Rica. ® All rights reserved. 
tratamiento fueron las quinolonas, tanto como monoterapia 0 en combinación, seguidas por los carbapenémicos y los glucopéptidos. Se utilizo en la mayoría de los casos terapias combinadas de distintos tipos de antibióticos.

Se utilizaron las herramientas estadísticas excel y el programa de estadística SPSS (Stadistical Program for the Social Sciences) V 15.0 , para tabular y graficar los datos obtenidos de la revisión de expedientes, esto con el fin de tener una mejor comprensión de los

\begin{abstract}
mismos. Se establecieron comparaciones entre los datos obtenidos en el estudio y los datos teóricos recopilados en la literatura como los dictados en las guías publicadas por la American Thoracic Society para el manejo de adultos con neumonía adquirida en la comunidad (ATS 2001) y para el manejo de adultos con neumonía adquirida en hospital y neumonía asociada a ventilador (ATS 2005), además de lo estipulado por la Guía Sanford de Tratamiento Antimicrobiano (2006), con el fin de cumplir con los objetivos planteados.
\end{abstract}

\section{Cuadro $\mathbf{N}^{\circ} 2$}

\section{Patógenos aislados en los cultivos de esputo ó aspirado bronquial}

\begin{tabular}{|c|c|c|}
\hline Patógeno & Número de & Porcentaje \\
\hline No identificado & 13 & 54.2 \\
Pseudomona aeruginosa & 1 & 4.2 \\
Moraxella sp. & 1 & 4.2 \\
Staphylococcus epidermidis & 1 & 4.2 \\
Pseudomona aeruginosa y K. pneumoniae & 1 & 4.2 \\
Enterobacter cloacae y Staphylococcus & 1 & 4.2 \\
aureus & 1 & 4.2 \\
Pseudomona aeruginosa y Staphylococcus & 1 & 4.2 \\
aureus & 1 & 4.2 \\
Staphylococcus epidermidis y Moraxella & 1 & 4.2 \\
sp. & 1 & 4.2 \\
Acinetobacter lwoffii, y Staphylococcus & 1 & 4.2 \\
\hline Klepidermidis & 1 & \\
Pseudomona aeruginosa y Escherichia coli & 1 & \\
Enterobacter cloacae & 1 & \\
\hline
\end{tabular}

Revista electrónica publicada por el Departamento de Farmacología de la Escuela de Medicina de la Universidad de Costa Rica, 2060 San José, Costa Rica. ® All rights reserved. 
Aún $83,3 \%$ de los pacientes se les realizó cambios a su antibioticoterapia inicial.

Sólo el $16,7 \%$ de los casos se mantuvo con la terapia inicial, el $58.3 \%$ presento como mínimo de 1 a 2 cambios en su tratamiento, y un $25 \%$ de las ocasiones se llegó a necesitar más tres cambios en su terapia antibiótica inicial. En las ocasiones que se aisló Pseudomona aeruginosa, ya sea solo o en combinación con otros microorganismos se necesito realizar más de un cambio en la medicación, inclusive en una ocasión necesito más de 6 cambios.

En un $75 \%$ de los casos las dosis eran correctas en las dosis de inicio de la terapia con antibióticos, en el restante $25 \%$ presentaron algunos errores, tanto por subdosificación como sobredosificación. Por otro lado, de los 20 casos en los cuales se realizaron cambios en la terapia antibiótica inicial, trece presentaban dosis correctas, los restantes siete presentaron al menos una dosis incorrecta.

\section{Cuadro $\mathbf{N}^{\circ} 3$}

Terapia inicial de los pacientes diagnosticados con neumonía

\begin{tabular}{|c|c|c|}
\hline Antibiótico & Número de & Porcentaje \\
\hline Cefalosporina 3 & Pacientes & \\
Quinolona & 2 & 8.3 \\
Carbapenémico & 3 & 12.5 \\
Glicopéptido & 1 & 4.2 \\
Aminoglicosido, Carbapenémico & 3 & 12.5 \\
Quinolona, Glicopéptido & 1 & 4.2 \\
Cefalosporina 3, Glicopéptido & 1 & 4.2 \\
Cefalosporina 3, Quinolona & 1 & 4.2 \\
Cefalosporina 4, Quinolona & 3 & 12.5 \\
Aminoglicosido, Quinolona, & 1 & 4.2 \\
Carbapenémico & 1 & 4.2 \\
Aminoglicosido, Carbapenémico, & 1 & 4.2 \\
Glicopéptido & 1 & 4.2 \\
Quinolona, Carbapenémico, & 1 & 4.2 \\
Glicopéptido & 1 & 4.2 \\
Carbapenémico, Glicopéptido & 1 & 4.2 \\
Carbapenémico, Glicopéptido, & 1 & 4.2 \\
Oxazolidinona & 1 & 4.2 \\
\hline Quinolona, Lincosamida & 1 & \\
Quinolona, Carbapenémico & 1 & \\
\hline
\end{tabular}

El número a la par del antibiótico tipo cefalosporina indica la generación de esta.

Revista electrónica publicada por el Departamento de Farmacología de la Escuela de 


\section{Cuadro $\mathbf{N}^{\circ} 4$}

Realización de prueba de sensibilidad a antibióticos

\section{Prueba de sensibilidad Número de Pacientes Porcentaje}

\begin{tabular}{l|c|c} 
No & 15 & 62.5 \\
$\mathrm{Si}$ & 9 & 37.5 \\
\hline
\end{tabular}

En el $37,5 \%$ de los casos se realizó prueba de sensibilidad a antibióticos para elegir la terapia. Tanto en los casos con NAC como en los de neumonía nosocomial se realizaron PSA en cantidades similares, las cuales fueron menores a los casos en los que no se realizaron PSA.

Existe una la alta resistencia a antibióticos generada por la bacteria $P$. aeruginosa, sobre todo si la comparamos con K. pneumoniae y Moraxella sp., según los datos obtenidos. Del total de pacientes diagnosticados con neumonía, seis fallecieron en el transcurso de la estancia hospitalaria; fallecieron más pacientes con diagnóstico de neumonía adquirida en la comunidad en comparación con los diagnosticados con neumonía nosocomial.

Tanto para los pacientes fallecidos como los que no, en la mayoría de sus casos el patógeno no fue diagnosticado. Para los pacientes no fallecidos el agente casual que predomino fue la Pseudomona aeruginosa. 


\title{
Cuadro $\mathbf{N}^{\circ} 5$
}

\section{Porcentaje de susceptibilidad a antibióticos de bacterias aisladas}

\author{
Antibiótico
}

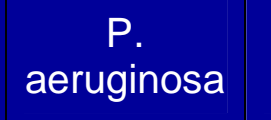

\begin{abstract}
E.
\end{abstract}

\begin{tabular}{c|c} 
K. & Moraxella \\
$\begin{array}{c}\text { pneumoni } \\
\text { ae }\end{array}$ & sp 1 \\
\hline
\end{tabular}

S.
aureus

S.

epidermidis

\begin{tabular}{|c|c|c|c|c|c|c|}
\hline Oxacilina & * & * & * & * & 50 & 33,3 \\
\hline Piperacilina & 57,1 & 100 & 50 & 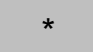 & * & * \\
\hline Piperacilina/Tazobactam & 57,1 & 100 & 50 & * & * & * \\
\hline Amoxicilina/ Clavulanato & 0 & * & * & 100 & 33,3 & 33,3 \\
\hline Penicilina G & * & * & * & * & 0 & 0 \\
\hline Ampicilina & 0 & 100 & 0 & 0 & 0 & 0 \\
\hline Ampicilina/Sulbactam & 0 & 100 & 50 & 0 & 0 & 0 \\
\hline Cefazolina & 0 & * & * & * & 33,3 & 100 \\
\hline Cefepime & 100 & 100 & 50 & 100 & 50 & 50 \\
\hline Cefotaxime & 0 & 100 & 50 & 100 & * & * \\
\hline Cefotetan & 0 & * & * & * & * & * \\
\hline Ceftazidima & 100 & 100 & 100 & * & * & 100 \\
\hline Ceftriaxona & 20 & 100 & 50 & 100 & 100 & 25 \\
\hline Cefaclor & 0 & * & 0 & 100 & * & * \\
\hline Cefixima & * & * & * & 50 & * & * \\
\hline Cefazolina & * & * & * & * & * & 0 \\
\hline Cefalexina & * & * & * & 100 & * & * \\
\hline Cefalotina & 0 & 50 & 50 & * & * & * \\
\hline Ertapenem & 0 & 100 & 100 & 100 & 60 & 0 \\
\hline Imipenem & 50 & 100 & 100 & * & 0 & * \\
\hline Meropenem & 42,9 & 100 & 100 & * & 50 & 50 \\
\hline Eritromicina & * & * & * & * & 50 & 33,3 \\
\hline Vancomicina & * & * & * & * & 100 & 100 \\
\hline Teicoplanina & * & * & * & * & 100 & 100 \\
\hline Gentamicina & 100 & 100 & 50 & * & 100 & 100 \\
\hline Amikacina & 85,7 & 100 & 50 & 50 & 100 & 100 \\
\hline Levofloxacina & 71,4 & 100 & 50 & 50 & 66,7 & 33,3 \\
\hline Moxifloxacino & 60 & 100 & 50 & 50 & 100 & 0 \\
\hline Ciprofloxacina & 71,4 & 100 & 50 & 50 & 50 & 33,3 \\
\hline Doxiciclina & * & * & 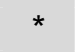 & 100 & 100 & 100 \\
\hline Rifampicina & * & * & * & * & 100 & 100 \\
\hline Linezolid & * & * & * & * & * & 100 \\
\hline TMP/SMX & 0 & 100 & 50 & 0 & 75 & 33,3 \\
\hline Clindamicina & * & * & * & * & 75 & 33,3 \\
\hline Tetraciclina & * & * & * & * & 80 & 100 \\
\hline
\end{tabular}

Color Rojo: Sólo se valoró un caso / * No se valoró

1 Moraxella sp, fueron dos los tipos de moraxellas analizadas y se decidió agruparlas.

Revista electrónica publicada por el Departamento de Farmacología de la Escuela de Medicina de la Universidad de Costa Rica, 2060 San José, Costa Rica. ® All rights reserved. 
En los casos de los fallecidos, para la terapia inicial se prefirió las combinaciones de antibióticos a la monoterapia, dichas combinaciones no fueron utilizadas en los pacientes que no fallecieron. Tanto en los casos de los pacientes fallecidos como los no fallecidos se sigue de forma predominante el patrón de realizar de 1 a 2 cambios a la terapia de inicio.

\section{Discusión}

La presente investigación analizó la antibiótico terapia utilizada y variables relacionadas de una población de 24 pacientes diagnosticados con neumonía adquirida en la comunidad o en el medio hospitalario. La edad de dicha población osciló entre los 48 y los 90 años siendo en su gran mayoría pacientes con más de 65 años, lo cual es uno de los factores de riesgo más importantes mencionados en la literatura para adquirir una neumonía en la comunidad, y además es uno de los criterios de ingreso hospitalario [3]. Sumado a lo anterior y estrechamente relacionado al mismo, las comorbilidades ocupan un sitio muy importante en el pronóstico del paciente con neumonía $[2,6]$.

Al igual que ocurre en la neumonía comunitaria, el diagnóstico microbiológico etiológico en la neumonía nosocomial es difícil, fundamentalmente por la colonización del tracto respiratorio superior que desarrollan los pacientes a lo largo de su ingreso, por los falsos positivos en el cultivo de esputo $u$ otras muestras y por la baja sensibilidad de los métodos invasivos para la obtención de muestras [2]. Así de los 12 casos a los cuales se les realizó cultivo, 11 identificaron alguna bacteria pero solo uno de ellos especificó cuál era dicha bacteria. Éste resultado concuerda con lo encontrado en la literatura pues la misma indica que entre un 40 y un $60 \%$ de los casos no se identifica el agente etiológico [5]. Los índices de infección polimicrobiana varían extensamente, pero los mismos van en aumento [3], la etiología polimicrobiana predominó en los casos analizados, siendo el patógeno más importante la Pseudomona aeruginosa, la cual también se aisló como patógeno único en uno de los casos analizados. La distribución de patógenos según el tipo de neumonía no guarda una clara relación, lo anterior debido al tamaño de la población; sin embargo se observa que entre los agentes que se determinaron como causa de neumonía adquirida en la comunidad, no se identificó al S. pnemoniae, considerado por American Thoracic Society como el principal causante de neumonía adquirida la comunidad que requiere hospitalización, seguido por $H$. influenzae, Legionella $s p$ y $S$. Aureus [7]. La terapia inicial es elegida de forma empírica, y según la literatura esta debería basarse en datos epidemiológicos, en el contexto en el que se adquirió la infección, presentación clínica y el conocimiento de patrones de sensibilidad actuales, además debe tener presente, el tiempo de hospitalización y los factores de riesgo para microorganismos multirresistentes [1]. Es importante resaltar que con respecto a esto, la terapia inicial, es tan variada que puede ir desde una sola Cefalosporina de tercera generación, hasta combinaciones de tres antibióticos entre los cuales se incluyen Carbapenémicos, Glucopéptidos y Oxazolidinonas. Se

Revista electrónica publicada por el Departamento de Farmacología de la Escuela de 
notó que los antibióticos más usados ya sean solos o en combinación con otros antimicrobianos son los Glucopéptidos (usados en 7 ocasiones), las Quinolonas [8], y por último los Carbapenémicos, los cuales participaron en las terapias de 9 de los 24 pacientes de la población analizada. Si bien los esquemas planteados por la American Thoracic Society establecen combinaciones de antibióticos, las combinaciones utilizadas en los casos estudiados no se apegan estrictamente a los mismos [3,7], aun así las terapias de inicio podrían erradicar el 0 los agentes causales según la epidemiología encontrada en la literatura.

La terapia inicial no se mantuvo en el $83,3 \%$ de los casos, eso corresponde a 20 de los 24 sujetos investigados, de este $83,3 \%$, la mayoría, corresponde a pacientes $(58,3 \%)$, los cuales recibieron solamente de uno a dos cambios en su medicación y en $25 \%$ de los casos se dieron más de tres modificaciones en la antibioticoterapia. Estos cambios de medicación incluyen tanto modificaciones de dosis, frecuencia ó cambio de antibióticos. Si bien la realización de estos cambios pudo haberse debido a la recepción de un cultivo con identificación de un patógeno

\section{CONCLUSIONES RECOMENDACIONES}

Al realizarse varios cambios a la estrategia antibiótica en la mayoría de los pacientes, se puede concluir que no se utilizan los datos epidemiológicos y las sensibilidades a los antibióticos, ni del hospital ni locales. La terapia prescrita podría colaborar entonces en un aumento de la estancia hospitalaria y resistencia de las bacterias a los antibióticos. Los antibióticos más específico o a la presencia de alguna otra enfermedad infecciosa que necesitara de eliminación mediante antibióticos, se tuvo claro durante el desarrollo de esta investigación que existe desconocimiento de la situación epidemiológica bacteriana tanto del hospital como local, así como de las sensibilidad bacteriana a los tratamientos antibióticos. Los resultados para P.aeruginosa encontrados en la unidad de cuidados intensivos indican mayor sensibilidad a quinolonas y a ciertas cefalosporinas en comparación con los datos obtenidos en el hospital en general, lo cual es contrario a lo que se esperaría encontrar según la literatura, pero dado a que la cantidad de pruebas de sensibilidad antibióticos realizadas es muy pequeña los datos obtenidos no son concluyentes.

De la población de 24 pacientes se reportaron 6 fallecimientos en la unidad de cuidados intensivos durante el periodo en estudio, 5 de los pacientes eran mayores de 70 años y como patología concomitante más comunes mostraban afecciones cardiacas. Ninguna de las combinaciones 0 monoterapias que recibieron los pacientes fallecidos les fue prescrita a los pacientes sobrevivientes.

utilizados son los glucopéptidos, quinolonas y carbapenémicos, usualmente utilizados en terapias combinadas que no necesariamente se ajustan a los esquemas de la American Thoracic Society.

En la institución se inicia a los pacientes diagnosticados con neumonía con una terapia inicial empírica de antibióticos, tal y como lo sugieren los organismos internacionales como la literatura consultada, sin embargo, la gran

Revista electrónica publicada por el Departamento de Farmacología de la Escuela de 
cantidad de cambios subsiguientes indican que la misma no es del todo efectiva. La mayoría de los patógenos que no fueron identificados requirieron cambios en la terapia inicial para su eliminación. Se recomienda investigar a cerca de los costos que tiene para institución la introducción como norma, de otros medios confirmatorios de diagnóstico para esta patología como lo son la radiografía de tórax y el cultivo de esputo 0 aspirado bronquial. Es imprescindible para el hospital, dar educación a los médicos sobre las consecuencias que puede acarrear para la institución el uso indiscriminado de antibióticos y las respectivas resistencias a estos fármacos localmente, con base en una serie de análisis más detallados de la

\section{Referencias}

1. Braunwald E, Fauci A, Dennis K, Longo D, Jameson L. Editores Harrison Principios de Medicina Interna. 15aㅡ ed. España. McGraw-Hill; 2001.

2. Abellán, G; Abizanda, P, Alastuey, C, et al. Tratado de Geriatría para Residentes. Madrid: Comité editorial Manuel Antón Jiménez [et al.]; 2007.

3. American Thoracic Society. Guidelines for the Management of adults with Hospital-acquired, Ventilator-associated Pneumonia. Am J Respir Crit Care Med. 2005; 171( ): 388-416.

4. Halm E; Teirstein A. Managament of Community-Acquired Pneumonia. N Engl Med. 2002; 347 (25): 2039-2045.

5. Álvarez L; Alós J; Blanquer J; Álvarez F. Guías para el manejo de la neumonía epidemiología bacteriana del hospital y sus diferentes unidades. Es importante establecer normas para el uso adecuado de los antibióticos, con el fin de darles un uso racional en pro de una disminución de la resistencia de las bacterias. En los expedientes no se lleva un orden claro, es común encontrar indicaciones medicas dictadas por teléfono, y es difícil encontrar las horas de aplicación de medicamentos por parte del personal de enfermería; se deben implementar normas para corregir esta situación con el afán de evitar alguna complicación legal. Se debe de tratar de concientizar al personal sobre la importancia del expediente médico como documento legal, y la ayuda que éste brinda en relación a la información importante. comunitaria del adulto que precisa ingreso en el hospital. Med Intensiva. 2005; 29(1): 21-62.

6. Fine M; Auble T; Yealy D; Hanusa B. A Prediction rule to identify low-risk patients with community-acquired pneumonia. N Engl Med. 1997; 336 (4): 243-250.

7. American Thoracic Society. Guidelines for the Management of adults with Community-acquired Pneumonia. Am J Respir Crit Care Med. 2001; 1730-1754.

8. Burgueño J. Uso de antibióticos en el paciente crítico sometido a ventilación mecánica. Aspectos relacionados con el tratamiento antibiótico de la neumonía asociada a ventilación mecánica. Facultad de medicina, Universidad Autónoma de Barcelona. España, 2005.

Revista electrónica publicada por el Departamento de Farmacología de la Escuela de 


\section{Correspondencia:}

\section{José Miguel Chaverri Fernández.}

Centro Nacional de Información de Medicamentos. Facultad de Farmacia. Universidad de Costa Rica. Apartado Postal 2060 San José, Costa Rica. Tel. 207-3476 Fax 207-5700.

Correo electrónico: imchaverri@cimed.ucr.ac.cr

ó imchaverri@farmacia.ucr.ac.cr 